\title{
Flexibility of representational states in working memory
}

\author{
Nahid Zokaei ${ }^{1,2}{ }^{*}$, Shen Ning ${ }^{1}$, Sanjay Manohar ${ }^{1,2}$, Eva Feredoes ${ }^{3}$ and Masud Husain ${ }^{1,2}$ \\ 'Department of Experimental Psychology, University of Oxford, Oxford, UK \\ ${ }^{2}$ Nuffield Department of Clinical Neurosciences, University of Oxford, Oxford, UK \\ ${ }^{3}$ School of Psychology and Clinical Language Sciences, University of Reading, Reading, UK
}

\section{Edited by:}

Jean-Philippe Van Dijck, Ghent

University, Belgium

Reviewed by:

Stephen Emrich, Brock University, Canada

Jyoti Mishra, University of California

San Francisco, USA

${ }^{*}$ Correspondence:

Nahid Zokaei, Department of Experimental Psychology,

University of Oxford, 9, South Parks

Road, Oxford, OX1 3UD, UK

e-mail: nahid.zokaei@psy.ox.ac.uk
The relationship between working memory (WM) and attention is a highly interdependent one, with evidence that attention determines the state in which items in WM are retained. Through focusing of attention, an item might be held in a more prioritized state, commonly termed as the focus of attention (FOA). The remaining items, although still retrievable, are considered to be in a different representational state. One means to bring an item into the FOA is to use retrospective cues ("retro-cues") which direct attention to one of the objects retained in WM. Alternatively, an item can enter a privileged state once attention is directed towards it through bottom-up influences (e.g., recency effect) or by performing an action on one of the retained items ("incidental" cueing). In all these cases, the item in the FOA is recalled with better accuracy compared to the other items in WM. Far less is known about the nature of the other items in WM and whether they can be flexibly manipulated in and out of the FOA. We present data from three types of experiments as well as transcranial magnetic stimulation (TMS) to early visual cortex to manipulate the item inside FOA. Taken together, our results suggest that the context in which items are retained in WM matters. When an item remains behaviorally relevant, despite not being inside the FOA, re-focusing attention upon it can increase its recall precision. This suggests that a non-FOA item can be held in a state in which it can be later retrieved. However, if an item is rendered behaviorally unimportant because it is very unlikely to be probed, it cannot be brought back into the FOA, nor recalled with high precision. Under such conditions, some information appears to be irretrievably lost from WM. These findings, obtained from several different methods, demonstrate quite considerable flexibility with which items in WM can be represented depending upon context. They have important consequences for emerging state-dependent models of WM.

Keywords: working memory, attention, representational states, retro cueing, incidental cueing

\section{INTRODUCTION}

Working memory (WM) refers to the ability to hold and manipulate information in mind for brief periods of time (Baddeley, 2003). It has been proposed that not all information in WM is maintained in an equal state. For example, depending on task relevance such as likelihood of being probed, one item might require prioritization over others. These sorts of considerations have led some authors to argue that the state of WM representations might be determined by an interaction between long-term memory (LTM) and attention (e.g., Cowan, 1998; McElree, 1998; Oberauer, 2002).

While these models differ in how the capacity and nature of representations in various states are determined (see Larocque et al., 2014 for a comprehensive review), they all agree on the existence of least two distinct states. One of these states has attention focused on an item (or a subset of items), rendering it in a more prioritized state so that it can be accessed more readily, with higher accuracy and/or fidelity (e.g., Lepsien and Nobre, 2007; Pertzov et al., 2012; Zokaei et al., 2014). In line with the most prominent theoretical models, we will refer to these prioritized items as being inside a focus of attention (FOA; as defined by Cowan, 1998; Oberauer, 2002, 2009). The remaining items outside FOA-although still retrievable-are considered to be in a different representational state (Figure 1).

The distinction between the items inside and outside FOA has been demonstrated both empirically (Lewis-Peacock et al., 2011; LaRocque et al., 2013; Stokes et al., 2013) and theoretically, often described in terms of the activated LTM proportion of short term memory (e.g., Oberauer, 2002). A substantial amount of research has focused on the qualities of representations of the item in FOA, but far less is known about the nature of the other items. One important but outstanding question that we address in this study is whether items can flexibly move in and out of FOA.

\section{MANIPULATION OF REPRESENTATIONAL STATES IN WM}

Behaviorally, the item in FOA is identified as the item that is recalled faster and more accurately compared to other retained items (see Nee and Jonides, 2013; Larocque et al., 2014 for 


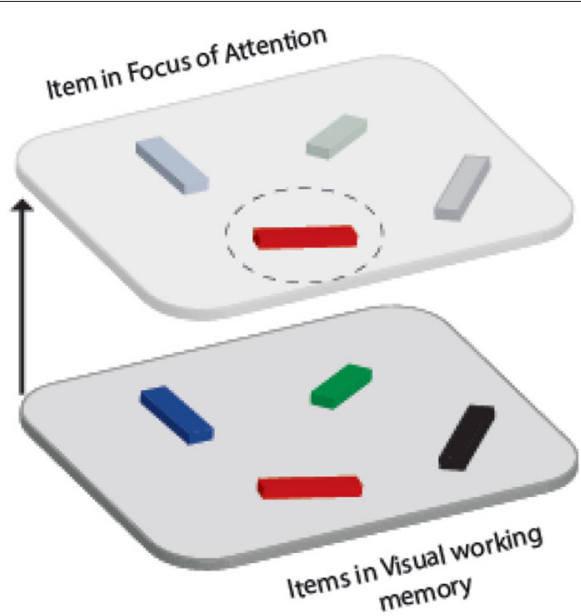

FIGURE 1 | Schematic representation of items maintained in visual WM in two different representational states. Although multiple items can be maintained in WM (lower panel), one item (red object in top pane) might be held in a more prioritized state known as the "focus of attention" (FOA).

examples). There are various methods for manipulating representational states in WM that result in such a behavioral advantage. In this section, we review selected studies that have used various approaches to shed light on the nature of the item in FOA and as a consequence on the other, non-focused items.

\section{Retro-cues shift the focus of attention in WM}

A commonly used means to bring an item into FOA is the use of retrospective cues ("retro-cues") presented during the retention interval, with the intention of directing attention to one specific item in WM. Specifically, a retro-cue will indicate to the participant which item is highly likely to be probed for recognition or recall. A consequence of retro cues is higher accuracy or precision of recall for the cued item compared to other uncued or invalidly cued items (Griffin and Nobre, 2003; Lepsien and Nobre, 2007; Makovsik and Jiang, 2007; Sligte et al., 2008, 2010; Astle et al., 2009; Lepsien et al., 2011; Berryhill et al., 2012; Pertzov et al., 2012).

Some studies have further attempted to characterize the neural underpinnings of the item in FOA achieved through retro-cueing (e.g., Lepsien and Nobre, 2007; Harrison and Tong, 2009; Lewis-Peacock et al., 2011; Nelissen et al., 2013; Larocque et al., 2014). In one such investigation, participants were presented with a sequence of two images to remember from two different categories of faces and scenes (Lepsien and Nobre, 2007). After a retention period they were shown a retro-cue indicating the relevant item for the upcoming memory probe. Event-related functional magnetic resonance imaging (fMRI) demonstrated that orienting attention to one of the items during WM maintenance modulated the activity in region of the brain involved in perception of the cued item: fusiform face area for faces and parahippocampal place area for scenes.

Using multi-voxel pattern analysis (MVPA; e.g., Haxby et al., 2001; Haynes and Rees, 2006) some investigators have reported stimulus-specific patterns in early visual areas during WM retention for retro-cued items. In a landmark study Harrison and Tong (2009) demonstrated that the orientation of the Gabor patch retro-cued during WM maintenance (from two retained orientations) could be decoded from the activity patterns in visual areas V1-V4. Nelissen et al. (2013) employed a similar retro-cueing WM task and showed that cued items could be accurately decoded from occipitotemporal cortex, but that decoding was at chance level for the uncued items (Nelissen et al., 2013; See also Lewis-Peacock et al., 2011).

From these findings one can conclude that the retro-cued item is maintained in a different state compared to non-cued items, with its maintenance dependent, at least to some extent, on early visual cortical regions known to be involved in perception of the maintained information.

\section{Recency: last item in the focus of attention}

Many investigations of WM present items serially rather than simultaneously. It has been known for a long time that the last item in a sequence is better recalled than previous ones: the recency effect. Some authors argue that the most recent item is in fact automatically in the FOA. Evidence comes from behavioral studies demonstrating retrieval advantage for the most recent item compared to items presented earlier in the sequence (McElree and Dosher, 1989, 1993; Neath, 1993; Hay et al., 2007; Blalock and Clegg, 2010). Furthermore, this last item is recalled faster, with more accuracy and precision compared to all previous items, with the magnitude of recency effect dependent on the number of previous items in the sequence (Gorgoraptis et al., 2011; Zokaei et al., 2011). Thus, the well-known recency effect in the WM literature might be due to the obligatory assignment of the last item to the FOA.

Brain imaging techniques have shed light on possible dissociation in neural correlates for maintenance of the last item compared to earlier items in a sequence. Using fMRI, Nee and Jonides (2008) investigated the probed-evoked neural signal of the most recently presented word compared to words presented earlier in a sequence. The recognition of the last item was accompanied by increased activation in the inferior temporal cortex. Similar findings have been presented in later studies (Nee and Jonides, 2011, 2013). Furthermore, recognition of the last word in the memory sequence was accompanied by less hippocampal activation when compared to recognition of all previous items in a sequence leading to intriguing suggestion that non-focused items are maintained by the hippocampus (Oztekin et al., 2009, 2010). Hence, there is both behavioral and neural evidence for dissociation of the most recent item into WM compared to previous items.

In addition recent findings using transcranial magnetic stimulation (TMS) have provided causal evidence for different representational states in visual WM (Zokaei et al., 2014). Two random dot kinematograms (RDKs) moving in different directions were presented sequentially, with the aim of bringing the second RDK into FOA. Following a delay period, participants had to recall the direction of one of the two previously-presented RDKs. Transcranial magnetic stimulation 
was administered during the delay of the WM task, prior to the presentation of the probe, to motion sensitive area MT+ which was hypothesized to be maintaining the remembered motion directions (Bisley and Pasternak, 2000; Pasternak and Greenlee, 2005). Transcranial magnetic stimulation impaired recall precision of the motion direction in FOA and, crucially, conversely improved precision for the other non-focused direction (Zokaei et al., 2014). These findings provide some of the first causal evidence that there are at least two representational states, with the maintenance of only the item in FOA relying on an area involved in its perception.

\section{Incidental-cueing brings an item into focus of attention}

A new method for manipulating representational states in WM has recently been developed; incidental cueing. This approach is different to the retro-cueing approach, because the cue is not explicitly predictive of upcoming memory recall. Instead, the rationale is that once an item from those maintained in WM is used for a cognitive operation that is incidental or orthogonal to the memory requirements of the task, that item will automatically enter the FOA.

In the study by Zokaei et al. (2014) participants were once again presented with two RDKs now simultaneously, above and below a fixation cross, and in two different colors. Participants were required to remember the directions of motion of each of the RDKs. During the delay period, the fixation cross changed to the color of one of the maintained motion directions and participants indicated with a key press the location of the motion direction of that color in the memory array, i.e., whether it had been above or below the fixation cross. The color of the fixation cross was not informative of the upcoming memory probe, and the judgment about the location of one of the RDKs was completely orthogonal to the requirement to remember the direction of motion of the RDKs. Nevertheless, adding this incidental task, resulted in higher precision of recall of the motion direction that also matched the color of the fixation cross. Thus, this item appeared to be in FOA simply by virtue of being "incidentally" cued. In agreement with the results from the TMS experiment described above, TMS applied here during the delay period, after the incidental cue, also disrupted recall of the cued item, and improved it for the noncued item.

A similar rationale was employed by Lewis-Peacock et al. (2011) who used a dual response and cueing WM task adapted from Oberauer (2005). Following a delay after presentation of the memory array, a cue appeared highlighting the first item to be probed. After the response, a second retro-cue appeared that indicated either the same item as that previously probed or alternatively cued the participant to switch to the other item in WM. For this second cue, only the task-relevant item (the cued item) could be successfully decoded from the fMRI BOLD signal, whereas the irrelevant (non-cued) item could not. This occurred despite no behavioral loss in performance when the second cue was different to the first (Lewis-Peacock et al., 2011, see LaRocque et al., 2013 for electroencephalography (EEG) analog).

The findings reported in this section provide evidence for the existence of a FOA within WM representations: an item can be held in a more privileged state with its recall more accurate and with higher precision compared to all other items maintained in WM. But what about the other items in WM? What happens to them and how are they stored?

\section{THE FATE OF ITEMS OUTSIDE THE FOCUS OF ATTENTION IN WM}

We now turn to investigations of how the items outside FOA are represented relative to those inside FOA. One hypothesis is that the item in FOA is protected from interference from un-cued items, with this protection coming at a cost for remembering un-cued items. Pertzov et al. (2012) simultaneously presented four oriented bars of different color and asked participants to remember their orientation. One of the bars was subsequently probed by its location or color. During the retention period, a retro-cue appeared indicating the item that was most likely to be later probed (70\% validity). In the subsequent recall period, participants were asked to reproduce the exact orientation of one of the bars. Precision of recall was significantly worse for the invalidly cued trials (i.e., trials where one of the un-cued items were probed) compared to trials in which the probe was validly cued, and also to baseline trials in which there was no cue.

These results were interpreted as evidence for the validly cued item being in a state that was protected from interference by other items held in WM, with these other objects suffering from accelerated temporal decay. Similar findings have been reported by Lepsien and Nobre (2007) and Matsukura et al. (2007). In the latter study, participants were presented with two consecutive retro-cues, with the second cue $100 \%$ valid. The second cue could be same or different to the first. Two identical cues resulted in similar performance to a single valid retro-cue, while two different cues impaired performance. The authors explained their findings in terms of forgetting un-cued items due to either degradation or interference, alongside protection of the cued item.

Evidence for the degradation of un-cued items is, however, inconsistent. In WM change detection tasks, several studies report no significant impairment in recall of the un-cued information with two consecutive cues during the delay (Landman et al., 2003; Rerko and Oberauer, 2013). For example, Rerko and Oberauer (2013) presented participants with a memory array followed by either a single, two or three cues. The probed matched $(50 \%$ trials) or mismatched the item that was cued last. There was no difference in accuracy between the cued and un-cued items.

The discrepancy in behavioral findings for memory of un-cued items may be explained by the degree of information conveyed by the cue. In tasks that show an effect on un-cued information (i.e., impaired performance in recalling those items) the cue carries predictive information indicating which will be the most relevant item-often the only one relevant itemfor forthcoming memory recall. As a consequence, un-cued information is rendered behaviorally irrelevant because of the low probability that it will need to be accessed again. In effect, this therefore changes the task to WM for a single (cued) item only.

On the other hand, in studies that have failed to find an effect on un-cued items (i.e., recall/recognition accuracy is no different to cued items), participants did not need to attend to previous cues, but rather the last cue only (e.g., Landman et al., 2003; Rerko and Oberauer, 2013). Thus, only the final cue was 
informative with respect to the upcoming probe, and importantly with only $50 \%$ validity. It could therefore be argued that given the low amount of predictive information conveyed by the cue, it was rendered less effective compared to retro-cues with, for example, $70 \%$ validity. Moreover, in these studies the final cue was followed by a very short delay $(<500 \mathrm{~ms})$ prior to the presentation of the probe. But for a retro-cue to produce sufficient behavioral advantage, there should be at least $1 \mathrm{~s}$ between the presentation of the cue and the memory probe (Pertzov et al., 2012).

\section{RECALL OF ITEMS NOT IN FOCUS OF ATTENTION}

From the studies described above it is evident that research has mainly centered on the single, privileged item in FOA. As a consequence, far less is known about the nature of non-focused items, and whether these items can be brought back into FOA or are lost from memory. In light of previous findings, one can hypothesize that whether items can flexibly move in and out of the FOA might be highly dependent on their potential relevance to the task in hand. Thus, if a non-focused item has a high chance of being probed it might still be maintained in a state such that it can be retrieved with high quality.

We tested this hypothesis across four experiments in which we used a method for measuring WM performance that relies on participants to reproduce the exact qualities of the retained information, providing a measure of precision of recall (Gorgoraptis et al., 2011; Zokaei et al., 2011, 2013). Such sensitive measure of WM allows us to detect small changes in recall precision that may otherwise not be detectable with alternative measures. In the first two experiments we used cues that were orthogonal to the WM task at hand. We hypothesized that these cues would allow items to move flexibly in and out of the FOA, since they carry no information regarding the relevance of items in WM. On the other hand, in experiment 3 and 4, a retro-cue with $80 \%$ validity was used. Unlike the first two experiments, we predicted that in such a situation, non-focused (un-cued) items in WM might degrade to the extent that they cannot be brought back into FOA.

In our first two experiments we aimed to examine whether representational states of items in WM can flexibly change in situations in which all items in WM remain potentially, behaviorally relevant throughout the trial. In Experiment 1 we used two successive incidental cues while in Experiment 2, we used sequential presentation of items so that the last item was in FOA by virtue of recency. This was then followed by an incidental cue that could be same or different to the last item in the sequence. Note that this method of cueing always required participants to make a response, allowing us to confirm whether they attended to the cued item or not, rather than relying on participants to attend to the cue of their own volition, with no objective measure (e.g., Landman et al., 2003; Pertzov et al., 2012; Rerko and Oberauer, 2013).

\section{EXPERIMENT 1: EFFECTS OF ONE OR TWO INCIDENTAL CUES METHODS \\ Participants}

Twenty healthy individuals (13 male) with an average age of 27 (range: 19-35) participated. All had normal or corrected to normal vision and reported normal color vision. They provided written consent to the procedure of the experiment, which was approved by the local ethics committee.

\section{Stimuli}

On each trial, two RDKs were presented below and above the fixation cross, subtending $10^{\circ}$ of visual angle. Each RDK consisted of 50 dots $\left(0.1^{\circ}\right.$ visual angle each $)$, displayed within an invisible circular aperture $\left(5.7^{\circ}\right.$ of visual angle). The color of the top RDK was chosen at random on each trial to be either green or red, with the lower RDK assigned the other color (red or green).

Dot lifetime and density were constant during RDK presentation with $100 \%$ coherent motion (constant speed of $4.5^{\circ} / \mathrm{s}$ ). Motion direction for each RDK was selected from $0-360^{\circ}$ with no angular separation between the two motion directions on each trial. A mask consisting of 5000 dots (50\% red), covering the entire screen was presented immediately after RDK offset.

Stimuli were displayed on 14.1" display (resolution $800 \times 600$ pixels, refresh rate $60 \mathrm{~Hz}$ ). Participants were seated approximately $60 \mathrm{~cm}$ from the monitor in a dimly lit room.

\section{Procedure}

A schematic representation of the task and different conditions is illustrated in Figure 2. Each trial started with a fixation cross (500 ms), followed by the presentation of two RDKs (the memory array for $300 \mathrm{~ms}$ ) and mask (100 ms). In this experiment, cues could be presented at two different time points, referred to as $1 \mathrm{st}$ position and 2 nd position cues.

In the baseline condition (1/9th of trials and not shown in the figure), the RDKs were simply followed by $7.2 \mathrm{~s}$ blank interval before presentation of probe stimuli. In 4/9th of trials, the stimuli were followed by a blank delay (1 s) and then the fixation cross briefly changed to either red or green (100 ms) which served as our method of incidental cueing. Participants had to indicate with a key press as accurately and as fast as possible the location of the RDK (above or below fixation cross) that was the same color as the fixation cross.

On half these trials (i.e., 2/9th of the overall trials), the cue was followed by a $6.1 \mathrm{~s}$ blank interval before the presentation of the probe (One cue, 1st condition-Figure 2A). On the remainder of the trials (2/9th of the overall number of trials), the memory array was followed by a $4.1 \mathrm{~s}$ delay, then the incidental cue (100 ms) and another delay ( $3 \mathrm{~s}$ ) before the presentation of the probe (One cue, 2nd position-Figure $2 \mathrm{~B})$.

In the remaining trials (4/9th of the overall trials), the cue was followed by 3 s delay before the presentation of a second incidental cue which could be the same (Figure 2C) or different color (Figure 2D) to the first cue (Two cues condition). Participants were informed that the cue was orthogonal to the memory task.

The memory probe was a circle $\left(5.7^{\circ}\right.$ visual angle in diameter $)$ presented at the center of the screen with a line from the center positioned at a randomly-selected orientation. On trials with one incidental cue and those with two identical colored cues, the color of the probe was the same (50\% of trials_congruent) or different (incongruent) to the color of the incidental cue(s).

In trials with two differently colored cues, on half the trials the probe was same color as the first cue while on the remaining the 


\section{A}
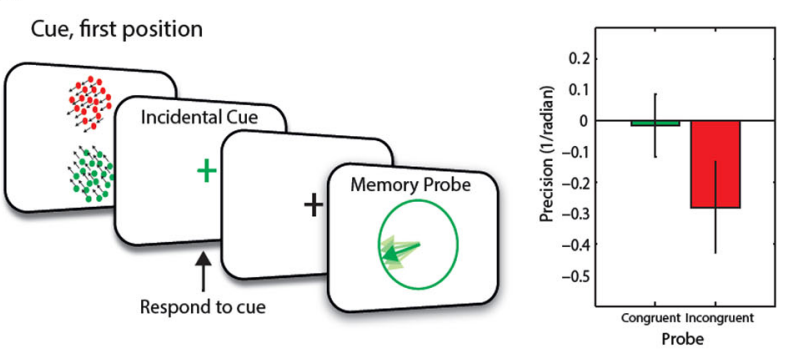

B

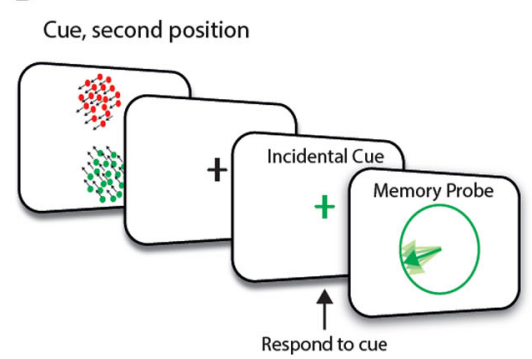

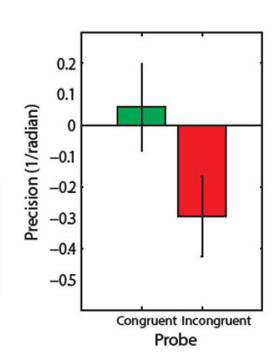

FIGURE 2 | Schematic and baseline corrected WM recall performance of trial events for Experiment 1. Two items were presented in the memory array. This was then followed by $(\mathbf{A})$ an incidental cue in the first position; (B)
C
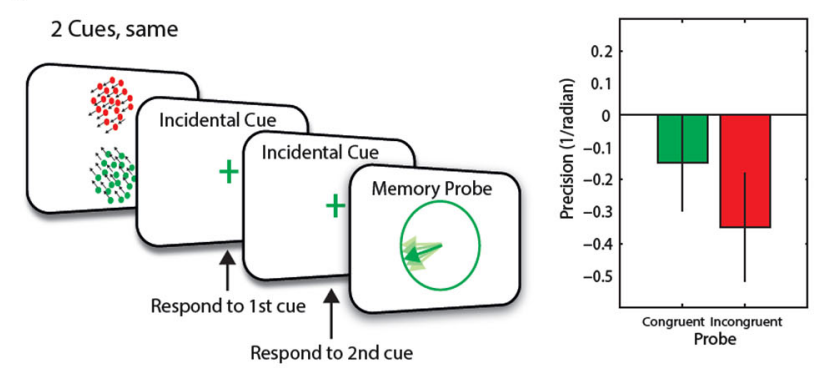

D

2 Cues, different
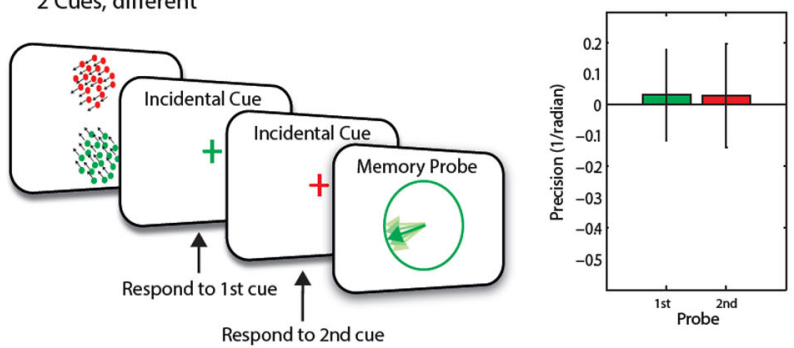

an incidental cue in the second position; (C) two identical consecutive incidental cues or (D) two different consecutive incidental cues, before the presentation of the memory probe. Error bars are SEM.

trials it was presented in the same color as the second cue. Using a mouse, participants adjusted the orientation of the line within the circle until it matched the direction of motion of the probed RDK. Accuracy of this matching procedure was emphasized over response time. Participants completed a practice block (30 trials) followed by seven blocks of 36 trials, different conditions randomly intermixed within a block.

\section{Precision calculation}

Recall error for the memory for motion task was calculated as the difference in response angle from target angle (i.e., the actual angle of the probed item). Recall precision was defined as the reciprocal of standard deviation of response error (Philipp, 2009). Note that precision is a measure of variability with higher precision corresponding to lower variability in memory. Due to small number of trials for all experiments reported here, we were unable to perform mixture modeling (Bays et al., 2009) to dissect our the sources of error resulting in a specific pattern of performance.

\section{RESULTS}

Participant performed the incidental cueing task with a high level of accuracy over all conditions (see Table 1 for mean accuracy and response times for all conditions). Trials in which there were incorrect responses to incidental cues were excluded from analysis.

We examined memory recall precision of motion direction for congruent vs. incongruent trials, corrected by performance in the baseline (no cue) condition in conditions where only one memory item was incidentally cued (i.e., the 1cue conditions, and two cues of the same color). There was an overall significant main effect of congruency, of whether the memory probe was about the same or different item to that specified by the previous incidental cue $\left(F_{(1,19)}=11.06, p=0.004\right)$. Thus recall precision was lower in the incongruent trials compared to congruent trials. This occurred in conditions when an incidental cue was presented in the first position $\left(t_{(19)}=3.7, p=0.001\right.$, Figure $\left.2 \mathrm{~A}\right)$, or second position (marginal significance, $t_{(19)}=1.8, p=0.084$, Figure 2B) or when two cues of the same color were presented $\left(t_{(19)}=2.27, p=0.035\right.$, Figure 2C). The incongruency effect for an incidental cue in the second position was marginally significant $\left(t_{(19)}=1.8, p=0.084\right.$, Figure 2B). These findings indicate successful incidental cueing. Performing the incidental task (which is orthogonal to the motion direction memory task) made a systematic difference to recall precision on the memory task, depending on congruency of incidental cue to the RDK that was later probed.

Importantly, the cueing advantage was apparently driven by a decrease in recall precision on incongruent trials, compared to congruent trials. Compared to no-cue baseline condition (zero in figures), there were marginally significant decreases in recall precision on incongruent trials when one cue occupied the 1st position $\left(t_{(19)}=1.89, p=0.07\right.$, Figure $\left.2 \mathrm{~A}\right)$ and when two cues of the same color were presented $\left(t_{(19)}=2.08, p=0.051\right.$, Figure 2C). There was a significant drop in recall precision for incongruent trials when one cue occupied the 2 nd position $\left(t_{(19)}=2.28\right.$, $p=0.035$, Figure 2B).

The critical condition, however, was when participants were presented with a sequence of two differently colored incidental cues. Note that in this case, we can consider the data in terms of whether the probe was the same as the item specified by the first incidental cue, or whether it was the same as the second 
Table 1 | Mean accuracy and response times for the two cues for all conditions (accuracy in percentage and response times in ms).

\begin{tabular}{|c|c|c|c|c|c|c|c|c|}
\hline Probe type & Same & Different & Same & Different & Same & Different & 1st item & 2nd item \\
\hline \multicolumn{9}{|l|}{ Cue 1} \\
\hline Accuracy (\%) & 83 & 87 & $\mathrm{n} / \mathrm{a}$ & $\mathrm{n} / \mathrm{a}$ & 85 & 87 & 89 & 86 \\
\hline RT (ms) & 742 & 730 & & & 715 & 723 & 730 & 722 \\
\hline \multicolumn{9}{|l|}{ Cue 2} \\
\hline Accuracy (\%) & $\mathrm{n} / \mathrm{a}$ & $\mathrm{n} / \mathrm{a}$ & 86 & 84 & 86 & 85 & 89 & 88 \\
\hline
\end{tabular}

cue. Prior to the second cue, one item would be considered to be in FOA. After it, however, another item might be brought into the FOA. This now becomes the "focused" item. Importantly recall precision for this item was neither significantly different to the no-cue/baseline $\left(t_{(19)}=0.2, p=0.85\right)$, nor to when a single cue was presented in 2nd position $\left(t_{(19)}=0.3, p=0.8\right.$; Figure 2D).

This finding shows that relatively high quality information regarding "other" items in WM-at least, similar to the baseline state-can be retrieved, in the context of a task where participants know that they might be asked to switch their attention between items in WM. This occurred despite the fact that such items were recalled with lower precision when they had not been brought into the privileged state of FOA by incidental cueing (Figures 2A-C).

Precision of recall for the "defocused" item in this condition, that is the item specified by the first cue and considered to be in FOA prior to the second cue was also no different than baseline $\left(t_{(19)}=0.2, p=0.83\right.$, Figure 2D) or to the item that was brought into FOA by the second cue $\left(t_{(19)}=0.02, p=0.99\right.$, Figure 2D). The lack of an effect on this item is discussed below.

\section{DISCUSSION}

These results demonstrate that in situations where items outside FOA remain potentially behaviorally relevant, they can be brought into FOA. The no cue, baseline condition used here allowed us to examine whether the effect of incidental cueing on WM performance was due to improvement of the item in FOA, or a degradation of the item outside it. The findings presented here show that the incidental cueing advantage can be explained specifically by a decrease in recall precision for the item outside FOA-rather than an advantage for the focused item-compared to baseline. So performing a task that requires consideration of the item specified by the incidental cue leads to a degradation of memory of the other item in WM, but no simultaneous boost to the cued item. Thus incidental cueing appears to operate in a very specific manner on the contents of WM.

A second important issue in these experiments is the fate of the de-focused item, that is the item that was inside FOA before the participant's attention was switched to another item as the trial progresses. Here, recall precision for the defocused item was comparable to baseline, as well as focused condition-but not worse. This might not be predicted on the basis of attention being drawn away from this item and instead raises the possibility that the capacity of FOA is larger than 1 item.

\section{EXPERIMENT 2: EFFECTS OF INCIDENTAL CUE ON RECENCY}

In the second experiment we aimed to examine whether representational states of items in WM can flexibly change in situations in which all items in WM remain potentially, behaviorally relevant throughout the trial using a different method. Here, we used sequential presentation of items so that the last item was in FOA by virtue of recency. This was then followed by an incidental cue that could be same or different to the last item.

\section{METHODS}

\section{Participants and stimuli}

Seventeen healthy individuals (10 male) with an average age of 25 (range: 20-31) participated in Experiment 2. On each trial, two RDKs, consisting ether of red or green moving dots, were now presented sequentially at screen center (Figure 3A), subtending $10^{\circ}$ of visual angle. Random dot kinematograms stimulus parameters were otherwise unchanged from Experiment 1.

\section{Procedure}

Each RDK was presented for $300 \mathrm{~ms}$ (+100 ms mask). There was 1000 ms blank delay between the two RDKs. In the baseline condition (not illustrated in the figure- $1 / 3$ of the overall trials), the 2 nd RDK was then followed by an unfilled delay of $4.1 \mathrm{~s}$. When cues were presented, the last RDK in the sequence was followed by a delay ( $1 \mathrm{~s}$ ) before the presentation of the incidental cue $(100 \mathrm{~ms})$, which matched the first RDK in $50 \%$ of the trials $(1 / 3$ of the overall trials) and the last item for the remaining trials. In this experiment, unlike the previous one, participants had to indicate with a key press the serial position (first or second) of the RDK with the same color as the incidental cue. There was a $3 \mathrm{~s}$ delay, which was then followed by the presentation of the probe.

The probe was in the same color as the first RDK on half of the trials and in the remaining trials, it matched the last item in the sequence. This therefore resulted in four experimental conditions with a $2 \times 2$ design with color of incidental cue as the first factor and probe color as the second factor, both with two levels: color matching the first or second item in the sequence. Participants completed a practice block (30 trials) followed by seven blocks of 30 trials, different conditions randomly intermixed within a block. 


\section{A}

Experiment 2: Task Schematic

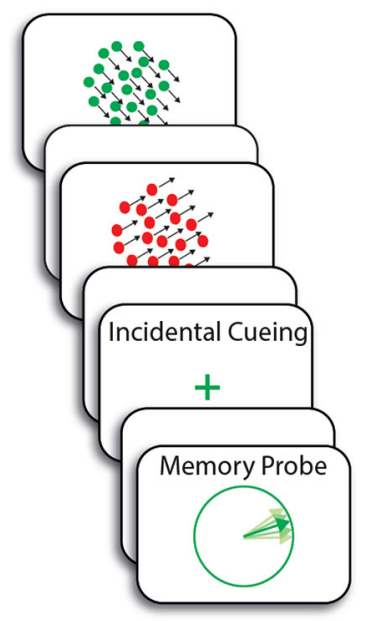

B

Experiment 2: Recall Precision

B.1) Cueing 1st item in the sequence

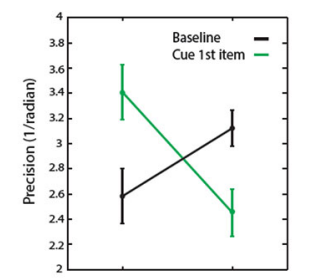

B.2) Cueing last item in the sequence

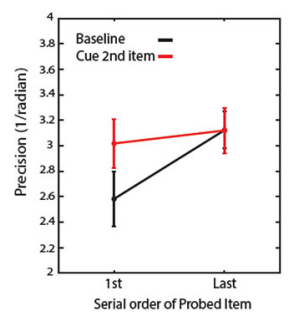

FIGURE 3 | (A) Schematic of trial events for Experiment 2. Following a sequential presentation of the memory array, the maintenance period was either blank or included an incidental cue before the presentation of the memory probe. (B) Recall precision for motion direction across conditions. Error bars are SEM.

\section{RESULTS}

Participant performed the incidental cueing task well, with mean accuracy of $95 \%(S D=5.3)$ and mean response time of $878 \mathrm{~ms}$ $(\mathrm{SD}=200 \mathrm{~ms})$. Trials with incorrect response in the incidental cueing task were excluded from further analysis.

We next investigated the effect of incidental cueing on precision of recall of the item in FOA compared to other items in WM. In the baseline condition, we obtained the long-established recency effect: the last item was recalled with greater precision than the first. In trials when the first item-presumed not be in the FOA by the end of the sequence-was later cued by the incidental cue, there was a significant interaction between cueing conditions (baseline, cue 1st item, cue last item) and serial order $\left(F_{(2,32)}=\right.$ 9.4, $p=0.001$ ).

Comparison of the baseline, no-cue condition to incidentally cueing the 1st item, revealed a significant interaction between cueing condition and serial order $\left(F_{(1,16)}=16.4, p=0.001\right.$, Figure 3B.1). This was due to an increase in recall precision for the first item in the sequence $\left(t_{(16)}=2.3, p=0.035\right)$-i.e., for the item now presumed to be in FOA-with a corresponding decrease in precision for the last, normally privileged, item $\left(t_{(16)}=2.8\right.$, $p=0.012$ ). This replicates our previous findings highlighting that in the context of an experiment where a non-privileged item in WM might be probed, it can be retrieved with relatively high precision after focusing attention upon it. In this experiment, unlike the first, we also found a cost in recall of the item that would otherwise be recalled with greater fidelity, here the last one in the sequence.

In trials where the last item in the sequence was cued, there was no significant interaction between cueing condition (cueing last item vs. baseline) and serial order of the probed item. The

last item could not be recalled with any greater precision than in the baseline condition. As for the first item, although there was a modest increase in recall precision, this was not significant $\left(t_{(16)}=1.8, p=0.087\right.$, Figure 3B.2). It is possible that the small increase in recall precision for the first item was due to rehearsal of items once the last item was cued, before making a response regarding its serial position. Participants might go through the sequence in their mind, resulting in refocusing on the first item before focusing on the last item in the sequence. In fact, response times to incidental cues of the last item in the sequence was marginally longer compared to trials where the 1st item in the sequence is cued $\left(t_{(16)}=1.9, p=0.078\right)$.

\section{DISCUSSION}

The results from Experiments 1 and 2 confirm our general hypothesis that in situations where items outside FOA remain potentially behaviorally relevant, they can be brought into FOA. In Experiment 2, precision of memory decreased for the last item (i.e., the item presumed to be in FOA) in trials where the incidental cue directed attention to the first item, which was now recalled with greater precision than baseline (Figure 3B.1). This simultaneous cost vs. benefit effect is different to that observed in Experiment 1 where recall precision for the defocused item was comparable to baseline, as well as focused condition. However, it is important to note that the effects of Experiment 1 were overall weaker (perhaps due to a much longer delay between encoding and probing of memory than in Experiment 2: $7.2 \mathrm{~s}$ vs. 4.1 ); this could possibly influence the lack of any effect of the de-focused item in the previous experiment.

The effects observed in Experiment 2 might be explained by two different mechanisms. It is possible that the capacity of the FOA is limited, perhaps to just one item, so that in trials where attention shifts to focus on another item in memory, the previously privileged item is displaced from FOA. However, this hypothesis would be inconsistent with some previous observations. Specifically, Rerko and Oberauer (2013) demonstrated an opposite effect for the defocused item than that reported here, with its recall significantly better compared to other items in WM for shorter delay periods.

Alternatively, it is possible that the time for which an item might be elevated to FOA by means of some types of cueing is limited. This is supported by EEG findings measuring temporal changes in item representation over the course of a delay period. LaRocque et al. (2013) employed an identical design to that of Lewis-Peacock et al. (2011) with EEG, demonstrating that approximately $1.25 \mathrm{~s}$ after cueing the unfocused item, classification accuracy for the initially cued item is decreased to levels comparable to baseline.

\section{RETRO-CUEING}

If the item in FOA is disrupted by either having attention switch to focus on another item in WM (Experiments 1 and 2) or artificially through TMS to early sensory areas (Zokaei et al., 2014), the item outside FOA can improve to levels comparable to the previously privileged item. However, as discussed previously, this is dependent on context: in the previous experiments reported above, non-FOA items remained relevant to the task 
in hand. Therefore, the question that remains is what happens to the other items in WM when they are rendered largely irrelevant?

Previous literature on this remains controversial (e.g., Landman et al., 2003; Lepsien and Nobre, 2007; Matsukura et al., 2007; Rerko and Oberauer, 2013). Due to differences in methodology comparison across studies to make meaningful general conclusions is difficult. To compare both the behavioral influences of re-focusing attention to other items in WM as well the causal effects of TMS, we conducted two retro-cueing investigations that are closely matched in timing and stimuli to two of our previous studies.

In Experiment 3 we used two successive cues, with the first one being a retro-cue, informative about the nature of the upcoming probe ( $80 \%$ validity). To ensure that participants attended to this cue, the second cue was either a stay or a switch cue, with $20 \%$ of the trials a switch cue. Importantly, this second cue was $100 \%$ valid, so participants knew for sure which item in WM was going to be probed. We aimed to investigate whether people can bring back the previously rendered irrelevant item following a switch trial. This experiment otherwise matched our double incidental cueing paradigm (Experiment 1) with two successive cues in timing and stimuli.

In Experiment 4 we used a similar retro-cue paradigm to that previously employed by Zokaei et al. (2014) (their Experiment 2) with an $80 \%$ valid rather than an incidental cue. Transcranial magnetic stimulation was administered during WM maintenance following the presentation of the cue to examine the causal role of early visual areas in maintenance of item in different representational states achieved through retro-cues and importantly its influence on the un-cued items.

\section{EXPERIMENT 3: STAY OR SWITCH FROM ITEM IN WM THAT WAS RETRO-CUED \\ METHODS \\ Participants}

Seventeen healthy individuals (14 Male) with an average age of 28 (range: 22-34) participated in Experiment 3. All had normal or corrected to normal vision and reported normal color vision. Participants provided written consent to the procedure of the experiment, approved by the local ethics committee.

\section{Procedure}

In each trial, similar to Experiment 1, two RDKs were presented above and below the fixation cross $(300 \mathrm{~ms}$ ) followed by a mask (100 ms; see Figure 4A). This was followed by a blank delay of $1000 \mathrm{~ms}$ prior to the presentation of the retro-cue, which was in the form of fixation cross color change $(100 \mathrm{~ms})$. The fixation cross changed from black either to red or green (50\% green) indicating the RDK that was more likely to be the cued later in the trial ( $80 \%$ validity for the next cue). This was then followed by a $3000 \mathrm{~ms}$ delay, before presentation of the second cue.

The second cue was $100 \%$ valid and was in the form of a word instructing participants to either "stay", i.e., maintain the same colored RDK as cued before ( $80 \%$ of trials) or "switch",

\section{A}

Experiment 3: Task Schematic

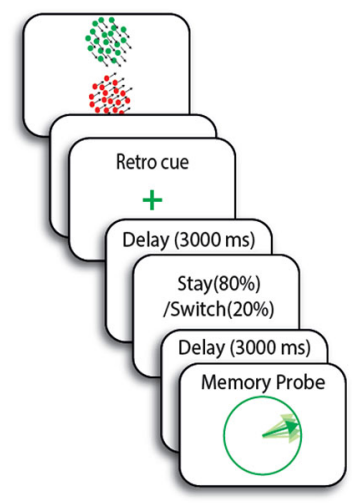

Experiment 3: Recall Precision

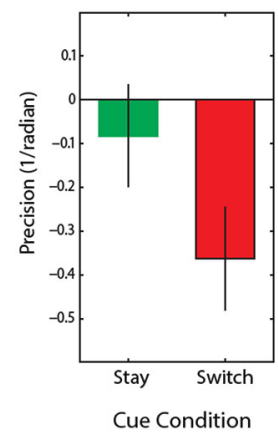

FIGURE 4 | (A) Schematic of trial events for Experiment 3. Memory array is followed by a retro-cue with $80 \%$ validity regarding the 2 nd cue. The second stay/switch cue was $100 \%$ valid for the upcoming probe. (B) Recall precision for the motion direction across conditions-baseline corrected. Error bars are SEM.

i.e., switch to the other item WM (20\% of trials). Note that in the vast majority of trials, therefore, the first retro-cue could be used to focus attention largely on one item held in WM.

Following a $3000 \mathrm{~ms}$ interval the item indicated by the second cue was probed and participants had to adjust the direction of the probe to match that of the cued item. These trials were intermixed with 30 baseline trials with no cueing during the delay but rather a long blank period $(7200 \mathrm{~ms}$ ) before presentation of the probe. Note that in this experiment, we used a switch/stay (second) cue in order to make sure participants attended to the first cue as no response to the that cue was required.

Participants completed a practice block (30 trials) followed by five blocks of 46 trials, different conditions randomly intermixed within a block and were informed of the validity of the cue prior to testing.

\section{RESULTS}

We first applied an ANOVA with cueing condition (valid, no-cue and invalid) as within-subject factors. There was a marginal effect of cue-type; $F_{(2,30)}=3.15, p=0.057$. Furthermore, recall precision was significantly higher in $(80 \%)$ stay compared to $(20 \%)$ switch trials $\left(t_{(14)}=2.7, p=0.0 .16\right.$; Figure $\left.4 \mathrm{~B}\right)$. With respect to the baseline (no-cue) condition, recall precision was not significantly different in stay trials $\left(t_{(14)}=0.8, p=0.4\right)$, but significantly decreased in switch trials $\left(t_{(14)}=2.45, p=0.027\right)$.

\section{DISCUSSION}

In this experiment the item that was initially retro-cued appeared to be held in a privileged state compared to the other item, which was effectively rendered largely irrelevant, because it was probed on only $20 \%$ of trials. These findings are consistent with the hypothesis that in situations where items outside FOA in WM 
are rendered largely irrelevant by the very low probability of being probed, they cannot subsequently be brought into FOA-at least to the level of precision of cued items - by having attention switched to them. The results are in line with previous experiments using informative cues to direct attention to one/subset of items in WM (e.g., Lepsien and Nobre, 2007; Matsukura et al., 2007; Pertzov et al., 2012). Next we used TMS to area MT+ in an attempt to disrupt the item in FOA.

\section{EXPERIMENT 4: EFFECTS OF TMS TO AREA MT+ METHODS \\ Participants}

Fifteen healthy individuals ( 8 Male) with average age of 25 (range: 18-32) participated in Experiment 4. All had normal or corrected to normal vision and reported normal color vision. Participants provided written consent to the procedure of the experiment, approved by the local ethics committee.

\section{MT+ localization and TMS}

A standard approach to MT+ localization using fMRI was applied (Huk et al., 2002). Left hemisphere clusters in the vicinity of MT+ (using anatomical guidelines described by Dumoulin et al., 2000) were identified in the native space of each participant and were overlaid onto their T1-weighted scan for a Brainsight frameless stereotaxy procedure (Rogue Research, Montreal, Canada). The participant's scalp location of left MT+ was marked on their scalp for subsequent TMS.

Stimulation was delivered via Magtism Rapid ${ }^{2}$ (The Magstim Company, Whitland, Wales, U.K.) using a 70-mm figure-eight coil. The coil handle pointed posteriorly rotated $45^{\circ}$, including a current approximately in the anterior or posterior direction. On each trial, 4 TMS pulses at $20 \mathrm{~Hz}$ were applied to left MT+ either at $60 \%$ ("high", effective intensity) or at $24 \%$ ("low", ineffective intensity) of maximum machine output. Low intensity trials were used to control for non-specific effects of TMS e.g., acoustic and tactile artifacts.

\section{Procedure}

Similar to Experiments 1 and 3, two RDKs were presented above and below fixation cross (300 ms) followed by a mask (100 ms; see Figure 5A) and blank delay $(1000 \mathrm{~ms})$. The fixation cross then changed briefly (similar to Experiment 3), out method of retro-cueing with $80 \%$ validity. This was then followed by a delay of $2600 \mathrm{~ms}$ before the administration of TMS. The TMS train lasted for $250 \mathrm{~ms}$ before presentation of the probe. The probe matched the color of the retro-cue in $80 \%$ of the trials (valid trials) while in the remaining $20 \%$ it did not (invalid trials). Trial sequence matched closely the timings of Zokaei et al. (2014) (their Experiment 2).

Participants completed a practice block (30 trials) followed by four blocks of 104 trials, different conditions randomly intermixed within a block.

\section{RESULTS}

In this experiment we compared high intensity (effective) TMS to low intensity (ineffective) TMS. Recall precision was significantly higher in valid (i.e., trials in which the probe matched the cue)

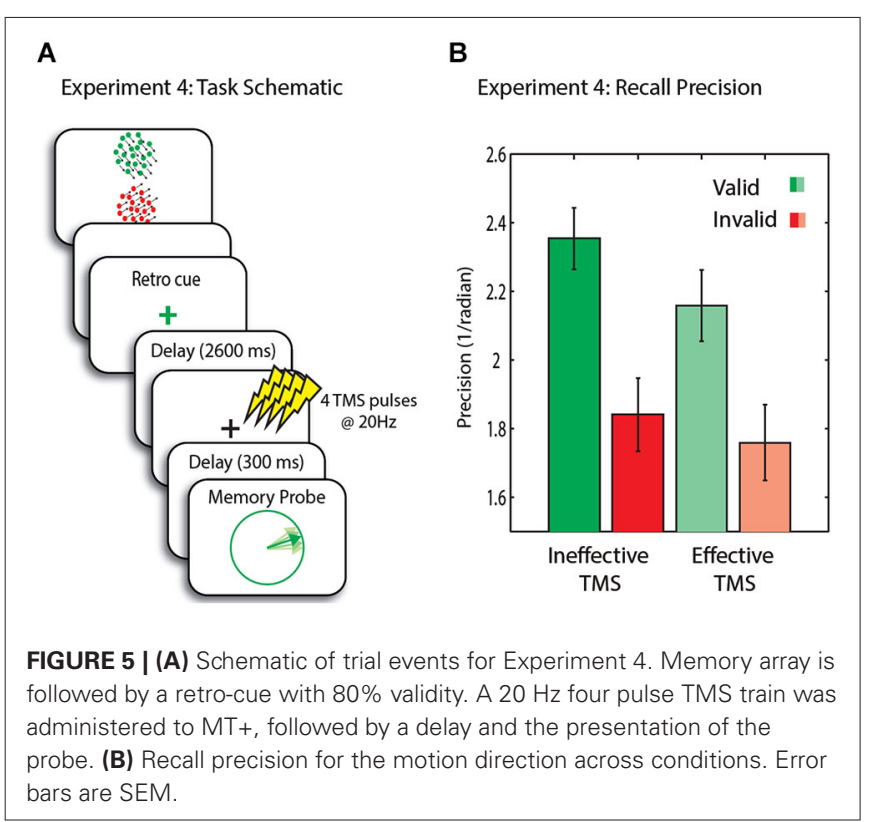

compared to invalid trials $\left(t_{(14)}=3.2, p=0.006\right)$, indicating successful retro-cueing (Figure 5B).

We next assessed whether high vs. low intensity TMS affected recall precision. A two-way ANOVA with factors TMS intensity and trial type yielded a significant main effect of validity $\left(F_{(1,14)}=9.5, p=0.008\right)$ and TMS intensity $\left(F_{(1,14)}=4.8\right.$, $p=0.045)$. The effect of TMS was due to a significant decrease in precision of memory after high intensity TMS on valid trials $\left(t_{(14)}=2.4, p=0.029\right.$, n.s after correcting for multiple comparisons), importantly with no change on invalid trials $\left(t_{(14)}=0.7, n . s.\right)$. Thus the privileged item sustained a cost with high intensity TMS. Nevertheless, the behavioral advantage of validity remained, with memory precision being significantly higher for valid compared to invalid trials $\left(t_{(14)}=2.3, p=0.039\right.$, n.s after correcting for multiple comparisons).

\section{DISCUSSION}

The results from the TMS experiment are consistent with the hypothesis that in situations where items outside FOA in WM are rendered largely irrelevant by the very low probability of being probed, they cannot be brought into FOA by disrupting the item in FOA with TMS. Here, we used an analogous procedure to that in our previous published study (Zokaei et al., 2014) with the only distinction of having a retro-cue rather than an incidental cue. To the best of our knowledge, that report is one of the first to investigate the causal role of early visual areas in maintenance of the retro-cued item in WM demonstrating that the maintenance of the cued item, in line with previous imaging studies (Lepsien and Nobre, 2007; Harrison and Tong, 2009; Nelissen et al., 2013), relies to some extent on visual areas involved in its perception (Zokaei et al., 2014).

In the current experiment, TMS to MT+ did not have an effect on the other item retained in WM, unlike the previously observed result with an incidental cue (Zokaei et al., 2014). In the 
study reported here, there was no improvement in recall precision for the other item in memory presumably because high quality information regarding this item was lost because of the extremely low $(20 \%)$ probability of being probed. It is, however, important to note that the smaller number of invalid relative to valid trials may also explain the lack of TMS effect on the invalid condition.

\section{GENERAL DISCUSSION}

The studies reported here sought to investigate the flexibility of representational states in WM: whether retained information can be flexibly moved in and out of the privileged state of FOA. Across four experiments, we explicitly and implicitly manipulated the state of two items in WM using different methods in order to place one in the FOA. Taken together, our results suggest that although the item in FOA is represented with higher recall precision compared to the non-privileged items in all experiments, the nature of the other items in WM crucially depends on the relevance of these items to the WM task. The context in which people are required to hold more than one item in WM makes a difference to how flexibly they can switch attention to non-focused items and improve their precision of recall. Also interesting to note is that the item in FOA was never recalled with greater precision than uncued items in the baseline condition. This suggests that the benefit conferred by the FOA is not necessarily due to straightforward increase in the precision with which the item is maintained compared to all other items. Indeed, this result fits with those of Gorgoraptis et al. (2011) in which recall precision of the last item in the sequence (i.e., the item in FOA) was similar to the precision of items when presented simultaneously regardless of memory set size. The implication is therefore that the FOA benefit may also depend on the context of the experiment.

In Experiment 1 we used either one or two consecutive incidental cues to explicitly bring the cued item into FOA: by requiring participants to make an action regarding a feature (in this case, location) of one of the retained items during WM delay period. The consequence of such incidental cueing is that this item, if probed later (congruent condition), will be recalled with higher precision than if the alternative item is probed (incongruent condition) (Zokaei et al., 2014). In Experiment 2 we used sequential presentation of the memory array followed by an incidental cue (in this case regarding, serial order), with the assumption that the last item in the sequence was in the privileged state by virtue of recency. Our working hypothesis, based on previous published studies discussed in the Section Introduction, is that the last cue in both these experiments places the cued item in the FOA. But importantly it carries no information regarding the relevance of the cued item for the upcoming WM task.

The results from these two studies demonstrated that in fact in situations where the item outside FOA remains potentially relevant to the task in hand, it can be brought back into the privileged state. Theoretically, these findings are in line with predictions made by the three-embedded components theory (Oberauer, 2002, 2009; Rerko and Oberauer, 2013) where no permanent detrimental effect on the other items in WM is predicted. In the context of this theoretical framework, the decrease in recall precision of the unfocused items might be due to direct interference from the item in FOA (e.g., Pertzov et al., 2012).
Thus abolishing such interference, either through disruptive effects of TMS (Zokaei et al., 2014) or by re-focusing attention on non-privileged items (as in Experiments 1 and 2 here), can increase their WM precision to levels comparable to baseline.

If the items outside FOA are indeed maintained and can be brought back into FOA, then what are the mechanisms underlying their retention? A few studies have demonstrated a role of the hippocampus in maintenance of items outside FOA, with their retrieval accompanied by activation in MTL (Oztekin et al., 2009, 2010; Nee and Jonides, 2011). Consistent with such observations, patients with hippocampal sclerosis have impaired WM performance only for items presented earlier in the sequence, that is items outside the FOA (López-Frutos et al., 2014). Alternatively, it has been suggested that the maintenance of unfocused items is supported either through sustained rapid short-term synaptic plasticity (Mongillo et al., 2008; Buonomano and Maass, 2009; Stokes et al., 2013) or sustained neuronal firing in non-sensory regions (Fuster and Alexander, 1971; Goldman-Rakic, 1995). However, support for each of these accounts is sparse and the brain mechanisms by which an item is brought into FOA have yet to be elucidated.

Unlike in the first two experiments, in Experiments 3 and 4 we manipulated representational states using retro-cues, i.e., cues that appear to focus attention on the most relevant item in WM for the upcoming probe. In Experiment 3, we used two consecutive cues: the first one was a retro-cue, informing participants on the likely nature of the second cue with $80 \%$ validity. The second cue was $100 \%$ valid for the WM probe. Recall precision for the invalidly cued WM item (i.e., the item probed on only $20 \%$ of trials) was significantly lower compared to the validly cued one, despite having attention switch to this item with a second $100 \%$ valid retro-cue. Therefore, the irrelevant item in WM following a retro-cue could not be brought back into FOA, despite having attention focus on it via the second retro-cue. Thus some information was, to some extent, irretrievably lost from WM following the initial retro-cue.

In Experiment 4, the precision of memory for the other/irrelevant items did not improve following disruptive effects of TMS to the item in FOA, unlike previous studies using incidental cueing or sequential presentation of items (Zokaei et al., 2014). However, while the probability of being probed regarding the invalid item was extremely low (20\%) in the TMS study we report here, in the incidental cueing study of Zokaei et al. (2014), there was a 50\% probability of being probed on the uncued item.

In that report, the improvement of memory for the nonFOA item in WM following TMS was described in terms of weakening of the interference from the item in FOA on the nonprivileged item. According to this hypothesis, TMS abolished the advantage of the item in FOA, leaving "baseline" WM performance intact and resulting in normalized performance for all items in WM. In accordance with such hypothesis, in the retro-cueing TMS study reported here (Experiment 4), "baseline" performance for the non-FOA items was decreased due to validity of the cue, prior to the administration of TMS. Abolishing the interference of item in the privileged state would therefore not be expected to improve the quality of the memory for the 
non-FOA item since it is maintained with low precision to begin with.

Findings from previous studies are not all consistent. Few have reported a cueing advantage (Lepsien and Nobre, 2007; Matsukura et al., 2007; Pertzov et al., 2012) while others have failed to observe one (Landman et al., 2003; Rerko and Oberauer, 2013). Current findings however can potentially explain such discrepancy in the literature if one considers the amount of information conveyed by the retro-cue regarding the likelihood of being probed on uncued items. In experiments that demonstrate a cueing advantage, the cue was valid, i.e., the cued item was later probed in majority of trials. On the other hand, in studies that have failed to report any effect of cueing, the cue was only 50\% valid and hence no different to a no cue condition. In addition, some studies using two consecutive cues have no method of confirming whether participants attended to the cues or not (e.g., Landman et al., 2003; Rerko and Oberauer, 2013), unlike the methods used here. Together these factors may well contribute to the inconsistency in the literature.

Our findings, in light of previous literature, suggest that the magnitude of cueing is dependent on the amount of information conveyed by the cue-the context in which WM holds items. Future research might aim to directly test this hypothesis by varying the validity of the cue systematically, although as the frequency of invalid cues is reduced the number of trials required to generate sufficient data would increase massively. Furthermore, current advances in modeling the sources of error associated with a specific pattern of performance may shed light on possible mechanisms that an item gains its privileged state (Bays et al., 2009). Similar findings with memory set sizes larger than two are also essential in building a comprehensive understanding of flexibility of representational states in WM.

The findings reported here also have important implications for both theoretical and neural computational models of WM. Although some have proposed dynamic representational states of items during WM retention (Fujisawa et al., 2008; Barak et al., 2010; Pascanu and Jaeger, 2011; Stokes et al., 2013), most models assume static representations (Seung and Sompolinsky, 1993; Lisman et al., 1998; Compte et al., 2000; Durstewitz et al., 2000; Mongillo et al., 2008; Dempere-Marco et al., 2012; Wei et al., 2012). Our results provide support for remarkable flexibility of representations in WM dependent upon context, and its close relationship to directed attention. Moreover, state-models of WM do not often make the distinction between other/non-privileged items in WM in different contexts, assuming a similar fate for all these items irrespective of how they have achieved their state. Our results provide strong empirical support for differential effects of cue validity-context of the WM task-on the nature of items outside FOA. Given the compelling evidence provided here, both computational and theoretical models of WM might profitably take into account dynamic representational states in WM when several items are retained.

\section{ACKNOWLEDGMENTS}

Research was supported by the Wellcome Trust (grant number: 098282).

\section{REFERENCES}

Astle, D. E., Scerif, G., Kuo, B.-C., and Nobre, A. C. (2009). Spatial selection of features within perceived and remembered objects. Front. Hum. Neurosci. 3:6. doi: 10.3389/neuro.09.006.2009

Baddeley, A. (2003). Working memory: looking back and looking forward. Nat. Rev. Neurosci. 4, 829-839. doi: 10.1038/nrn1201

Barak, O., Tsodyks, M., and Romo, R. (2010). Neuronal population coding of parametric working memory. J. Neurosci. 30, 9424-9430. doi: 10.1523/jneurosci. $1875-10.2010$

Bays, P. M., Catalao, R. F. G., and Husain, M. (2009). The precision of visual working memory is set by allocation of a shared resource. J. Vis. 9, 7.1-7.11. doi: $10.1167 / 9.10 .7$

Berryhill, M. E., Richmond, L. L., Shay, C. S., and Olson, I. R. (2012). Shifting attention among working memory representations: testing cue type, awareness and strategic control. Q. J. Exp. Psychol. (Hove) 65, 426-438. doi: 10.1080/17470218. 2011.604786

Bisley, J. W., and Pasternak, T. (2000). The multiple roles of visual cortical areas MT/MST in remembering the direction of visual motion. Cereb. Cortex 10, 1053-1065. doi: 10.1093/cercor/10.11.1053

Blalock, L. D., and Clegg, B. A. (2010). Encoding and representation of simultaneous and sequential arrays in visuospatial working memory. Q. J. Exp. Psychol. (Hove) 63, 856-862. doi: 10.1080/17470211003690680

Buonomano, D. V., and Maass, W. (2009). State-dependent computations: spatiotemporal processing in cortical networks. Nat. Rev. Neurosci. 10, 113-125. doi: $10.1038 / \mathrm{nrn} 2558$

Compte, A., Brunel, N., Goldman-Rakic, P. S., and Wang, X. J. (2000). Synaptic mechanisms and network dynamics underlying spatial working memory in a cortical network model. Cereb. Cortex 10, 910-923. doi: 10.1093/cercor/10.9.910

Cowan, N. (1998). Attention and Memory: An Integrated Framework. NY: OUP.

Dempere-Marco, L., Melcher, D. P., and Deco, G. (2012). Effective visual working memory capacity: an emergent effect from the neural dynamics in an attractor network. PLoS One 7:e42719. doi: 10.1371/journal.pone.0042719

Dumoulin, S. O., Bittar, R. G., Kabani, N. J., Baker, C. L. Jr., Le Goualher, G., Bruce Pike, G., et al. (2000). A new anatomical landmark for reliable identification of human area V5/MT: a quantitative analysis of sulcal patterning. Cereb. Cortex 10, 454-463. doi: 10.1093/cercor/10.5.454

Durstewitz, D., Seamans, J. K., and Sejnowski, T. J. (2000). Neurocomputational models of working memory. Nat. Neurosci. 3, 1184-1191. doi: 10.1038/81460

Fujisawa, S., Amarasingham, A., Harrison, M. T., and Buzsáki, G. (2008). Behaviordependent short-term assembly dynamics in the medial prefrontal cortex. Nat. Neurosci. 11, 823-833. doi: 10.1038/nn.2134

Fuster, J. M., and Alexander, G. E. (1971). Neuron activity related to short-term memory. Science 173, 652-654. doi: 10.1126/science.173.3997.652

Goldman-Rakic, P. S. (1995). Cellular basis of working memory. Neuron 14, 477485. doi: 10.1016/0896-6273(95)90304-6

Gorgoraptis, N., Catalao, R. F. G., Bays, P. M., and Husain, M. (2011). Dynamic updating of working memory resources for visual objects. J. Neurosci. 31, 85028511. doi: 10.1523/jneurosci.0208-11.2011

Griffin, I. C., and Nobre, A. C. (2003). Orienting attention to locations in internal representations. J. Cogn. Neurosci. 15, 1176-1194. doi: 10.1162/ 089892903322598139

Harrison, S. A., and Tong, F. (2009). Decoding reveals the contents of visual working memory in early visual areas. Nature 458, 632-635. doi: 10.1038/ nature 07832

Haxby, J. V., Gobbini, M. I., Furey, M. L., Ishai, A., Schouten, J. L., and Pietrini, P. (2001). Distributed and overlapping representations of faces and objects in ventral temporal cortex. Science 293, 2425-2430. doi: 10.1126/science.1063736

Hay, D. C., Smyth, M. M., Hitch, G. J., and Horton, N. J. (2007). Serial position effects in short-term visual memory: a SIMPLE explanation? Mem. Cognit. 35, 176-190. Available online at: http://eprints.lancs.ac.uk/11411/. [Accessed on July 8, 2011]. doi: 10.3758/bf03195953

Haynes, J.-D., and Rees, G. (2006). Decoding mental states from brain activity in humans. Nat. Rev. Neurosci. 7, 523-534. doi: 10.1038/nrn1931

Huk, A. C., Dougherty, R. F., and Heeger, D. J. (2002). Retinotopy and functional subdivision of human areas MT and MST. J. Neurosci. 22, 7195-7205.

Landman, R., Spekreijse, H., and Lamme, V. A. F. (2003). Large capacity storage of integrated objects before change blindness. Vision Res. 43, 149-164. doi: 10 . 1016/s0042-6989(02)00402-9 
LaRocque, J. J., Lewis-Peacock, J. A., Drysdale, A. T., Oberauer, K., and Postle, B. R. (2013). Decoding attended information in short-term memory: an EEG study. J. Cogn. Neurosci. 25, 127-142. doi: 10.1162/jocn_a_00305

Larocque, J. J., Lewis-Peacock, J. A., and Postle, B. R. (2014). Multiple neural states of representation in short-term memory? It's a matter of attention. Front. Hum. Neurosci. 8:5. doi: 10.3389/fnhum.2014.00005

Lepsien, J., and Nobre, A. C. (2007). Attentional modulation of object representations in working memory. Cereb. Cortex 17, 2072-2083. doi: 10.1093/ cercor/bhll16

Lepsien, J., Thornton, I., and Nobre, A. C. (2011). Modulation of working-memory maintenance by directed attention. Neuropsychologia 49, 1569-1577. doi: 10 . 1016/j.neuropsychologia.2011.03.011

Lewis-Peacock, J. A., Drysdale, A. T., Oberauer, K., and Postle, B. R. (2011). Neural evidence for a distinction between short-term memory and the focus of attention. J. Cogn. Neurosci. 24, 61-79. doi: 10.1162/jocn_a_00140

Lisman, J. E., Fellous, J.-M., and Wang, X.-J. (1998). A role for NMDA-receptor channels in working memory. Nat. Neurosci. 1, 273-275. doi: 10.1038/1086

López-Frutos, J. M., Poch, C., García-Morales, I., Ruiz-Vargas, J. M., and Campo, P. (2014). Working memory retrieval differences between medial temporal lobe epilepsy patients and controls: a three memory layer approach. Brain Cogn. 84, 90-96. doi: 10.1016/j.bandc.2013.11.004

Makovsik, T., and Jiang, Y. V. (2007). Distributing versus focusing attention in visual short-term memory. Psychon. Bull. Rev. 14, 1072-1078. doi: 10. 3758/bf03193093

Matsukura, M., Luck, S. J., and Vecera, S. P. (2007). Attention effects during visual short-term memory maintenance: protection or prioritization? Percept. Psychophys. 69, 1422-1434. doi: 10.3758/BF03192957

McElree, B. (1998). Attended and non-attended states in working memory: accessing categorized structures. J. Mem. Lang. 38, 225-252. doi: 10.1006/jmla.1997. 2545

McElree, B., and Dosher, B. A. (1989). Serial position and set size in short-term memory: the time course of recognition. J. Exp. Psychol. Gen. 118, 346-373. doi: 10.1037/0096-3445.118.4.346

McElree, B., and Dosher, B. A. (1993). Serial retrieval processes in the recovery of order information. J. Exp. Psychol. Gen. 122, 291-315. doi: 10.1037/0096-3445. 122.3.291

Mongillo, G., Barak, O., and Tsodyks, M. (2008). Synaptic theory of working memory. Science 319, 1543-1546. doi: 10.1126/science.1150769

Neath, I. (1993). Distinctiveness and serial position effects in recognition. Mem. Cognit. 21, 689-698. doi: 10.3758/bf03197199

Nee, D. E., and Jonides, J. (2008). Neural correlates of access to short-term memory. Proc. Natl. Acad. Sci. U S A 105, 14228-14233. doi: 10.1073/pnas.080208 1105

Nee, D. E., and Jonides, J. (2011). Dissociable contributions of prefrontal cortex and the hippocampus to short-term memory: evidence for a 3-state model of memory. Neuroimage 54, 1540-1548. doi: 10.1016/j.neuroimage.2010. 09.002

Nee, D. E., and Jonides, J. (2013). Trisecting representational states in short-term memory. Front. Hum. Neurosci. 7:796. Available online at: http://www.ncbi.nlm.nih.gov/pmc/articles/PMC3840432/ [Accessed on April 3, 2014]. doi: 10.3389/fnhum.2013.00796

Nelissen, N., Stokes, M., Nobre, A. C., and Rushworth, M. F. S. (2013). Frontal and parietal cortical interactions with distributed visual representations during selective attention and action selection. J. Neurosci. 33, 16443-16458. doi: 10. 1523/jneurosci.2625-13.2013

Oberauer, K. (2002). Access to information in working memory: exploring the focus of attention. J. Exp. Psychol. Learn. Mem. Cogn. 28, 411-421. doi: 10. 1037//0278-7393.28.3.411

Oberauer, K. (2005). Binding and inhibition in working memory: individual and age differences in short-term recognition. J. Exp. Psychol. Gen. 134, 368-387. doi: 10.1037/0096-3445.134.3.368

Oberauer, K. (2009). "Chapter 2 design for a working memory," in Psychology of Learning and Motivation, ed B. H. Ross (USA: Academic Press),
45-100. Available online at: http://www.sciencedirect.com/science/article/pii/ S007974210951002X. [Accessed on May 10, 2013].

Oztekin, I., Davachi, L., and McElree, B. (2010). Are representations in working memory distinct from representations in long-term memory? Neural evidence in support of a single store. Psychol. Sci. 21, 1123-1133. doi: 10. $1177 / 0956797610376651$

Oztekin, I., McElree, B., Staresina, B. P., and Davachi, L. (2009). Working memory retrieval: contributions of the left prefrontal cortex, the left posterior parietal cortex and the hippocampus. J. Cogn. Neurosci. 21, 581-593. doi: 10.1162/jocn. 2008.21016

Pascanu, R., and Jaeger, H. (2011). A neurodynamical model for working memory. Neural Netw. 24, 199-207. doi: 10.1016/j.neunet.2010.10.003

Pasternak, T., and Greenlee, M. W. (2005). Working memory in primate sensory systems. Nat. Rev. Neurosci. 6, 97-107. doi: 10.1038/nrn1603

Pertzov, Y., Bays, P. M., Joseph, S., and Husain, M. (2012). Rapid forgetting prevented by retrospective attention cues. J. Exp. Psychol. Hum. Percept. Perform. 39, 1224-1231. doi: 10.1037/a0030947

Philipp, B. (2009). CircStat: a MATLAB toolbox for circular statistics. J. Stat. Softw. Rerko, L., and Oberauer, K. (2013). Focused, unfocused and defocused information in working memory. J. Exp. Psychol. Learn. Mem. Cogn. 39, 1075-1096. doi: 10. 1037/a0031172

Seung, H. S., and Sompolinsky, H. (1993). Simple models for reading neuronal population codes. Proc. Natl. Acad. Sci. U S A 90, 10749-10753. doi: 10. 1073/pnas.90.22.10749

Sligte, I. G., Scholte, H. S., and Lamme, V. A. F. (2008). Are there multiple visual short-term memory stores? PLoS One 3:e1699. doi: 10.1371/journal.pone. 0001699

Sligte, I. G., Vandenbroucke, A. R. E., Scholte, H. S., and Lamme, V. A. F. (2010). Detailed sensory memory, sloppy working memory. Front. Psychol. 1:175. doi: 10.3389/fpsyg.2010.00175

Stokes, M. G., Kusunoki, M., Sigala, N., Nili, H., Gaffan, D., and Duncan, J. (2013). Dynamic coding for cognitive control in prefrontal cortex. Neuron 78, 364-375. doi: 10.1016/j.neuron.2013.01.039

Wei, Z., Wang, X.-J., and Wang, D.-H. (2012). From distributed resources to limited slots in multiple-item working memory: a spiking network model with normalization. J. Neurosci. 32, 11228-11240. doi: 10.1523/jneurosci.0735-12. 2012

Zokaei, N., Gorgoraptis, N., Bahrami, B., Bays, P. M., and Husain, M. (2011). Precision of working memory for visual motion sequences and transparent motion surfaces. J. Vis. 11:2. doi: 10.1167/11.14.2

Zokaei, N., Heider, M., and Husain, M. (2013). Attention is required for maintenance of feature binding in visual working memory. Q. J. Exp. Psychol. (Hove) 67, 1191-1213. doi: 10.1080/17470218.2013.852232

Zokaei, N., Manohar, S., Husain, M., and Feredoes, E. (2014). Causal evidence for a privileged working memory state in early visual cortex. J. Neurosci. 34, 158-162. doi: 10.1523/jneurosci.2899-13.2014

Conflict of Interest Statement: The authors declare that the research was conducted in the absence of any commercial or financial relationships that could be construed as a potential conflict of interest.

Received: 28 July 2014; accepted: 03 October 2014; published online: 06 November 2014.

Citation: Zokaei N, Ning S, Manohar S, Feredoes E and Husain M (2014) Flexibility of representational states in working memory. Front. Hum. Neurosci. 8:853. doi: 10.3389/fnhum.2014.00853

This article was submitted to the journal Frontiers in Human Neuroscience.

Copyright ( 2014 Zokaei, Ning, Manohar, Feredoes and Husain. This is an open-access article distributed under the terms of the Creative Commons Attribution License (CC BY). The use, distribution and reproduction in other forums is permitted, provided the original author(s) or licensor are credited and that the original publication in this journal is cited, in accordance with accepted academic practice. No use, distribution or reproduction is permitted which does not comply with these terms. 